\title{
Electronic Absorption Spectra of Some Triazolopyrimidine Derivatives
}

\author{
Hussein Moustafa, ${ }^{1}$ M. F. Shibl, ${ }^{1}$ Rifaat Hilal, ${ }^{1}$ Laila I. Ali, ${ }^{2}$ and Sheimaa Abdel Halim² \\ ${ }^{1}$ Chemistry Department, Faculty of Science, Cairo University, Giza 12613, Egypt \\ ${ }^{2}$ Chemistry Department, Faculty of Education, Ain Shams University, Cairo 12613, Egypt
}

Correspondence should be addressed to Rifaat Hilal, rhhilal@hotmail.com

Received 2 December 2010; Revised 22 January 2011; Accepted 24 February 2011

Academic Editor: Linda B. McGown

Copyright (C) 2011 Hussein Moustafa et al. This is an open access article distributed under the Creative Commons Attribution License, which permits unrestricted use, distribution, and reproduction in any medium, provided the original work is properly cited.

\begin{abstract}
The electronic absorption spectra of triazolo pyrimidine and some of its derivatives were measured in polar as well as nonpolar solvents. Assignment of the observed transitions is facilitated via molecular orbital calculations. Charge density distributions, dipole moments, and the extent of delocalization of the MOS were used to interpret the observed solvent effects. The observed transitions are assigned as charge transfer (CT), localized, and delocalized according to the contribution of the various configurations in the CI-states. The correspondence between the calculated and experimental transition energies is satisfactory.
\end{abstract}

\section{Introduction}

Pyrimidine and fused heterocyclic pyrimidine derivatives have attracted a great deal of interest due to their biochemical and pharmaceutical activities. Triazolopyrimidines, in particular, were tested for their medicinal, bactericidal, and fungicidal activity [1-3]. Wide variety of interesting biological activities were observed for those compounds, such as anticancer [4], antiviral [5], anti-H1V-1 activity [6], antiinflammatory [7] and antimicrobial activities [8]. In addition, several substituted dihydronaphthothieno pyrimidine derivatives showed antimicrobial activities against Bacillus subtilis, Escherichia coli, Aspergillus niger, and Candida albicans [9], and their ester-containing derivatives demonstrated more antimicrobial activities than the corresponding cyanocontaining analogs.

The UV spectra of pyrimidines have been investigated [10]. The spectrum, in general, depends on the state of the nucleus, whether it is fully aromatic, partly reduced, or fully reduced and on the position, number, and nature of any chromophoric substituent attached to it. Furthermore, it also depends greatly on presence of ionic species in solution being measured. The UV absorption spectra of pyrimidines show two main band systems centered at 243 and $298 \mathrm{~nm}$ in cyclohexane. The second band is ascribed to an $n-\pi^{*}$ transition on account of the hypsochromic shift observed as changing solvent from cyclohexane to water. The lone pair become engaged in H-bonding in water so that the absorbed radiation must be of higher energy, that is, of lower wavelength. Loakes et al. $[11,12]$ studied the spectra of some triazolopyrimidines and discussed the effect of varying the acidity of the medium on the observed spectra. Effect of alkyl substitution on the spectra of some triazolopyrimidines was recently investigated [13] qualitatively.

A detailed series of MO studies at the HMO level have been carried out on purines, pyrazolopyrimidines, triazolopyrimidines, and pyrazolopyrimidines [14-16]. The aim of such qualitative MO calculation is to ascertain the extent of aromaticity in the studied compounds. More recently, the electronic structure of a model triazolopyrimidine structure has been performed at the RHF and DFT levels of theory [17]. This study aimed at exploring the electronic structure of platinum complexes of triazolopyrimidines. A similar high level calculation has also been reported for the Ruthenium triazolopyrimidine complexes [18].

In part I of this work [19], the ground state geometries of some Triazolopyrimidines were optimized at the density 


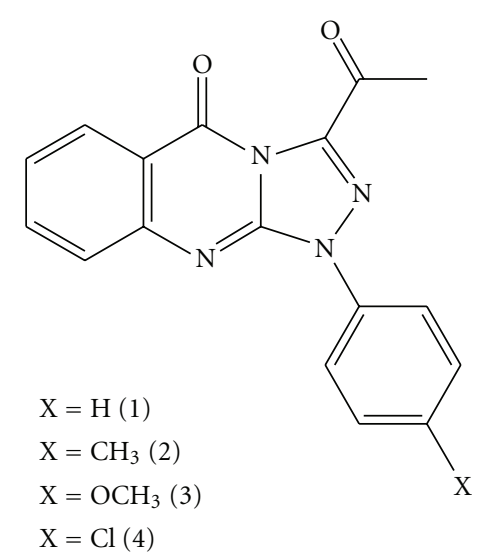

Scheme 1

functional level of theory (DFT). Molecular structures were fully optimized using B3LYP/6-31G basis set. The effects of substituents on the geometry and electronic structural features of triazolo pyrimidine were examined.

The electronic structure of molecules usually manifests itself in the electronic absorption and emission spectra. This manifestation enables the detailed understanding of the forces that govern the electronic structure of the studied Triazolopyrimidines. Although several investigations have been published that dealt with the electronic spectra of pyrimidines, yet there is no systematic study of substituents and solvent effects on the observed spectra. Such study is of critical importance in understanding their electronic structure that underlines their biological activity.

The aim of our paper is to explore (1) the type and extent of conjugative interaction between different subsystems of Triazolopyrimidines, (2) the effect of solvent polarity on the observed spectra and, hence, predict the relative stabilities, extent of charge transfer character, and assignment of the observed electronic transitions, and (3) the effect of para-phenyl substitution, using electron-donating and withdrawing groups on the observed electronic spectra of Triazolopyrimidines.

\section{Experimental}

2.1. Compounds. Compounds studied were prepared and purified by standard procedures cited in the literature [20] see Scheme 1.

2.2. Solvents. Polar (methanol) and nonpolar (dioxane) solvents were obtained from Merck, AR-grade and were used without any further purification.

2.3. Apparatus. Spectra were scanned on a Perkin Elmer lambda $4 \mathrm{~B}$ spectrophotometer using $1.0 \mathrm{~cm}$ fused silica cells. The machine records linearly in percent transmittance over the range 190-900 $\mathrm{nm}$.

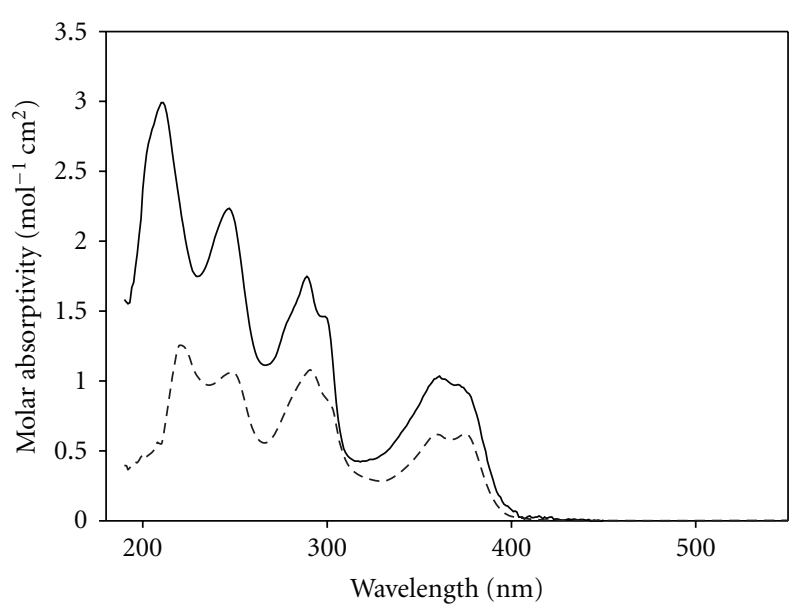

- Methanol

- - - Dioxane

FIGURE 1: Electronic absorption spectra of (1).

\section{Method of Calculation}

Molecular orbital calculations, employing full geometry optimization, were performed on the ground states of the molecules studied, at the DFT- B3LYP/6-31G [21, 22] level of theory using Gaussian .03 program [23]. Excited states were treated theoretically using ZINDO/S [24].

\section{Result and Discussion}

4.1. The Electronic Absorption Spectra of (1). The electronic absorption spectra in nonpolar (Dioxane) and polar (methanol) solvents are presented in Figure 1. The charge density maps of the occupied and vacant MOs considered in the present analysis are presented in Figure 5. Tables 1 and 2 present a comparison of the experimentally observed and theoretically computed spectra in addition to a quantitative assignment of all transitions observed.

The spectrum, in dioxane, is composed of three main band systems. The long wavelength system is composed of two bands centered at 375 and $360 \mathrm{~nm}$ and the medium envelope composed of three bands centered at 325, 301, and $298 \mathrm{~nm}$. The third band system is composed of two bands centered at 250 and $223 \mathrm{~nm}$. Increasing solvent polarity causes blue shift of all observed bands. Furthermore, increasing solvent polarity causes a marked decrease in the intensity of all bands. All the observed bands may be assigned to $\pi-\pi^{*}$ transitions as reflected from their intensities (4000-30000). This is in general agreement with previously published analysis of the spectra of triazoles [25].

Excited configurations, considered in this paper, are those which result from an electron excitation between the highest three occupied molecular orbitals $\Psi_{54}-\Psi_{56}$ and the lowest three unoccupied molecular orbitals $\Psi_{57}-\Psi_{59}$. The configuration interaction matrix is solved, and the configuration interaction state functions are given in Table 1. 
TABle 1: Calculated and observed band maxima, intensities, and dipole transition of 3-Acetyl-1-phenyl-1H-[1, 2, 4] triazolo [3, 4-b] quinazolin-5-one.

\begin{tabular}{|c|c|c|c|c|c|c|c|}
\hline \multirow{3}{*}{ State } & \multicolumn{5}{|c|}{ Theoretical } & \multicolumn{2}{|c|}{ Experimental } \\
\hline & Configuration & Coefficient & $\lambda, \mathrm{nm}$ & $f$ & $\mu$ & Polar & Nonpolar \\
\hline & & & & & & $\lambda, \mathrm{nm}$ & $\lambda, \mathrm{nm}$ \\
\hline \multirow{2}{*}{ I } & $56-57$ & 0.5193 & 382 & 0.001 & 5.6759 & 375 & 375 \\
\hline & $56-58$ & 0.8130 & & & & & \\
\hline II & $56-57$ & 0.870 & 350 & 0.558 & 0.9529 & 362 & 360 \\
\hline \multirow[t]{2}{*}{ III } & $56-57$ & 0.912 & 320 & 0.7622 & 2.5338 & 320 & 325 \\
\hline & $56-59$ & 0.646 & 300 & 0.1449 & 5.5869 & 300 & 301 \\
\hline \multirow[t]{2}{*}{ IV } & $55-57$ & 0.503 & & & & & \\
\hline & $54-57$ & 0.520 & & & & & \\
\hline $\mathrm{V}$ & $56-59$ & 0.864 & 286 & 0.0437 & 13.6527 & 289 & 298 \\
\hline VI & $54-57$ & 0.775 & 252 & 0.2082 & 8.5341 & 248 & 250 \\
\hline \multirow{2}{*}{ VII } & $55-58$ & 0.472 & 223 & 0.0466 & 5.0933 & 212 & 223 \\
\hline & $54-58$ & 0.759 & & & & & \\
\hline
\end{tabular}

TABLE 2: State energies, configurations, type of transition, and assignment of all transitions for 3-Acetyl-1-phenyl-1H-[1, 2, 4] triazolo [3, 4-b] quinazolin-5-one.

\begin{tabular}{lcccc}
\hline State & Configuration & Type & Assignment & Obs. \\
\hline I & $56-57$ & $\pi-\pi^{*}$ & Delocalized band & 3.31 \\
& $56-58$ & $\pi-\pi^{*}$ & Delocalized band & 3.25 \\
II & $56-57$ & Delocalized band & 3.44 \\
III & $56-57$ & $\pi-\pi^{*}$ & Delocalized band & 3.54 \\
& $56-59$ & $\pi-\pi^{*}$ & CT: N- aryl $\rightarrow$ Triazolo-pyrimidine & 3.88 \\
IV & $55-57$ & $\pi-\pi^{*}$ & CT: N- aryl $\rightarrow$ Triazolo-pyrimidine & 4.12 \\
& $54-57$ & CT: N- aryl $\rightarrow$ Triazolo-pyrimidine & 4.13 \\
V & $56-59$ & $\pi-\pi^{*}$ & Delocalized band & 4.16 \\
VI & $54-57$ & $\pi-\pi^{*}$ & CT: N- aryl $\rightarrow$ Triazolo-pyrimidine & 4.34 \\
VII & $55-58$ & $\pi-\pi^{*}$ & CT: N- aryl $\rightarrow$ Triazolo-pyrimidine & 4.92 \\
& $54-58$ & $\pi-\pi^{*}$ & CT: N- aryl $\rightarrow$ Triazolo-pyrimidine & 5.56 \\
\hline
\end{tabular}

The long wave length absorption band has been computed theoretically at $382 \mathrm{~nm}$ and composed of two configurations, namely, $\phi_{56}^{-1} \phi_{57}$ and $\phi_{56}^{-1} \phi_{58}$. The configuration $\phi_{56}^{-1} \phi_{57}$ represents $27 \%$ of this state whereas $\phi_{56}^{-1} \phi_{58}$ represents $66 \%$. Both configurations are delocalized.

The second $\left(\pi, \pi^{*}\right)^{1}$ state is computed theoretically at $350 \mathrm{~nm}$ which is in good agreement with the experimentally observed value of $360 \mathrm{~nm}$. This state is composed of one configuration, namely, $\phi_{56}^{-1} \phi_{57}$, and possesses the same polarity as the ground state, hence, shows no pronounced solvent effect.

The third $\left(\pi, \pi^{*}\right)^{1}$ state is computed theoretically at $320 \mathrm{~nm}$ and composed of one configuration, $\phi_{56}^{-1} \phi_{57}$. This band shows a blue shift on increasing solvent polarity, that is, solvent dependent. This is maybe attributed to the relatively high polarity of the excited state as compared to that of the ground state. This state may be assigned as a delocalized transition.
The fourth $\left(\pi, \pi^{*}\right)^{1}$ state is computed theoretically at $300 \mathrm{~nm}$. This state is composed of three configurations, namely, $\phi_{56}^{-1} \phi_{59}, \phi_{55}^{-1} \phi_{57}$, and $\phi_{54}^{-1} \phi_{57}$. This state shows no solvent dependence and assigned as a charge transfer band (CT) from $\mathrm{N}$-aryl to triazolo pyrimidine moiety.

The fifth $\left(\pi, \pi^{*}\right)^{1}$ state computed at $298 \mathrm{~nm}$ in dioxane, $289 \mathrm{~nm}$ in methanol and is computed theoretically at $286 \mathrm{~nm}$. This state possesses a high polarity as compared to that of the ground state $(\approx 13.6 \mathrm{D})$, and hence solvent dependence on band position is expected. This state is also assigned as a delocalized transition.

The last two states computed theoretically appear at $252 \mathrm{~nm}$ and $223 \mathrm{~nm}$. Both states are $\pi-\pi^{*}$ and assigned as CT bands from $\mathrm{N}$-aryl to triazolo pyrimidine (c.f. Tables 1 and 2).

4.2. The Electronic Absorption Spectra of (2). Figure 2 presents the electronic absorption spectra of (2) in polar 
TABLE 3: Calculated and observed band maxima, intensities, and dipole transition of 3-Acetyl-1-p-tolyl-1H-[1, 2, 4] triazolo [3, 4-b] quinazolin-5-one.

\begin{tabular}{|c|c|c|c|c|c|c|c|}
\hline \multirow{3}{*}{ State } & \multicolumn{5}{|c|}{ Theoretical } & \multicolumn{2}{|c|}{ Experimental } \\
\hline & Configuration & Coefficient & $\lambda, \mathrm{nm}$ & $F$ & $\mu$ & Polar & Nonpolar \\
\hline & & & & & & $\lambda, \mathrm{nm}$ & $\lambda, \mathrm{nm}$ \\
\hline \multirow{2}{*}{ I } & $59-60$ & 0.813 & 371 & 0.5612 & 5.2449 & 375 & 376 \\
\hline & $59-61$ & 0.484 & & & & & \\
\hline II & $59-60$ & 0.897 & 326 & 0.8362 & 4.1182 & 340 & 325 \\
\hline III & $59-61$ & 0.895 & 274 & 0.5813 & 8.5551 & 275 & 275 \\
\hline \multirow{2}{*}{ IV } & $58-61$ & 0.556 & 224 & 0.0273 & 4.9168 & 220 & 225 \\
\hline & $57-61$ & 0.653 & & & & & \\
\hline
\end{tabular}

TABLE 4: State energies, configurations, type of transition, and assignment of all transitions for 3-Acetyl-1-p-tolyl-1H-[1, 2, 4] triazolo [3, 4-b] quinazolin-5-one.

\begin{tabular}{lcccr}
\hline State & Configuration & Type & Assignment & \multicolumn{1}{c}{$\begin{array}{c}\text { Obs. } \\
\text { Calc. }\end{array}$} \\
\hline I & $59-60$ & $\pi-\pi^{*}$ & Delocalized band & 3.34 \\
& $59-61$ & $\pi-\pi^{*}$ & Delocalized band & 3.30 \\
II & $59-60$ & $\pi-\pi^{*}$ & Delocalized band & 3.82 \\
III & $59-61$ & $\pi-\pi^{*}$ & Delocalized band & 4.51 \\
IV & $58-61$ & $\pi-\pi^{*}$ & CT: N- aryl $\rightarrow$ Triazolo-pyrimidine, & 5.51 \\
& $57-61$ & $\pi-\pi^{*}$ & CT: N- aryl $\rightarrow$ Triazolo-pyrimidine & 5.54 \\
\hline
\end{tabular}

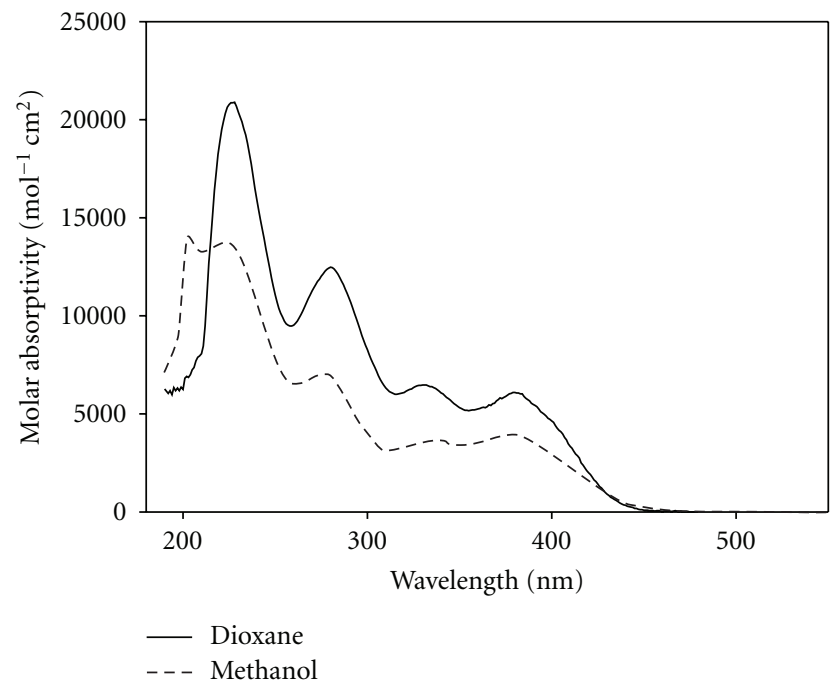

FIGURE 2: Electronic absorption spectra of (2).

as well as nonpolar solvents. The spectrum, in nonpolar solvent, shows an envelope that covers the area 320$380 \mathrm{~nm}$ and contains two bands centered at $376 \mathrm{~nm}$ and $325 \mathrm{~nm}$, and another envelope covers the area $220-280 \mathrm{~nm}$ showing two main bands centered at $275 \mathrm{~nm}$ and $225 \mathrm{~nm}$. Increasing solvent polarity decreases the intensity of all observed bands with a red shift of the long wavelength
$325 \mathrm{~nm}$ band in dioxane to $340 \mathrm{~nm}(\approx 15 \mathrm{~nm})$. The three occupied MOs $\psi_{57}-\psi_{59}$ and two vacant MOs $\psi_{60}-\psi_{61}$ are considered in the CI computations. Tables 3 and 4 compare the experimentally observed and theoretically computed transitions, assignment, configurations involved and their coefficients. The $\left(\pi, \pi^{*}\right)^{1}$ states observed experimentally are well reproduced theoretically. All the observed transition are $\pi-\pi^{*}$ as indicated from their intensities (4000-20000).

The first $\left(\pi, \pi^{*}\right)^{1}$ is transition centered at $371 \mathrm{~nm}$ is composed of two configurations, namely, $\psi_{59}^{-1} \psi_{60}$ and $\psi_{59}^{-1} \psi_{61}$. Both configurations are assigned as delocalized bands.

The second $\left(\pi, \pi^{*}\right)^{1}$ computed theoretically at $326 \mathrm{~nm}$ and composed of only one configuration, $\psi_{59}^{-1} \psi_{60}$. This state is much more polar than the ground state and is expected to show solvent dependence $(\approx 15 \mathrm{~nm})$.

The last two bands are computed theoretically at $274 \mathrm{~nm}$ and $224 \mathrm{~nm}$ which agree with the experimentally observed spectra in both polar and nonpolar solvents. The band at $274 \mathrm{~nm}$ may be assigned as a delocalized transition whereas the band at 224 is assigned to a CT transition from the Naryl moiety to the triazolo pyrimidine subsystem (cf. Tables 4 and 5).

4.3. The Electronic Absorption Spectra of (3). Figure 3 presents the electronic spectra of (3) in methanol and dioxane as solvents. The spectrum in dioxane is composed of two long wavelength bands centered at $355 \mathrm{~nm}$ and $313 \mathrm{~nm}$ and two short wavelength bands centered at $263 \mathrm{~nm}$ and 
TABLE 5: Calculated and observed band maxima, intensities, and dipole transition of 3-Acetyl-1-(4-methoxy-phenyl)-1H-[1, 2, 4] triazolo [3, 4-b] quinazolin-5-one.

\begin{tabular}{lccccccc}
\hline State & \multicolumn{3}{c}{ Theoretical } & & & \multicolumn{2}{c}{ Experimental } \\
& Configuration & Coefficient & $\lambda, \mathrm{nm}$ & $F$ & $\mu$ & $\begin{array}{c}\text { Polar } \\
\lambda, \mathrm{nm}\end{array}$ & $\begin{array}{c}\text { Nonpolar } \\
\lambda, \mathrm{nm}\end{array}$ \\
\hline I & $62-63$ & 0.8505 & 361 & 0.5234 & 5.0213 & 368 & 355 \\
II & $62-64$ & 0.7953 & 326 & 0.3086 & 10.1508 & 312 & 313 \\
III & $61-63$ & 0.839 & 263.5 & 0.3584 & 10.6888 & 262 & 263 \\
IV & $61-63$ & 0.845 & 228 & 0.0543 & 15.9894 & 227 & 225 \\
\hline
\end{tabular}

TABLE 6: State energies, configurations, type of transition, and assignment of all transitions for 3-Acetyl-1-(4-methoxy-phenyl)-1H-[1, 2, 4] triazolo [3, 4-b] quinazolin-5-one.

\begin{tabular}{lccccr}
\hline State & \multirow{2}{*}{ Configuration } & Type & Assignment & Obs. & \multicolumn{1}{c}{ Calc. } \\
\hline I & $62-63$ & $\pi-\pi^{*}$ & Delocalized band & 3.49 & 3.43 \\
II & $62-64$ & $\pi-\pi^{*}$ & Delocalized band & 3.96 & 3.80 \\
III & $61-63$ & $\pi-\pi^{*}$ & CT: N- aryl $\rightarrow$ Triazolo-pyrimidine & 4.71 & 4.71 \\
IV & $61-63$ & $\pi-\pi^{*}$ & CT: N- aryl $\rightarrow$ Triazolo-pyrimidine & 5.51 & 5.44 \\
\hline
\end{tabular}

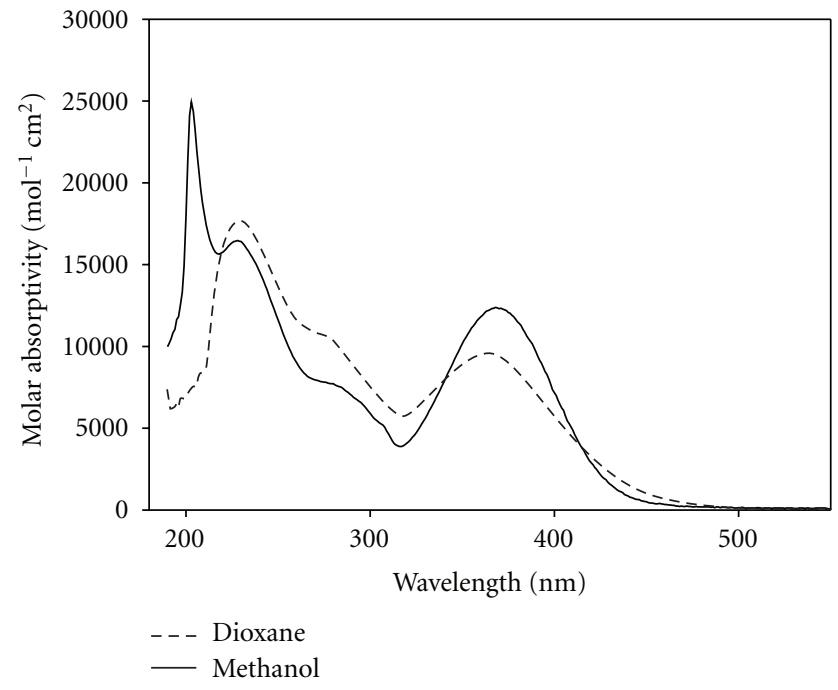

Figure 3: Electronic absorption spectra of (3).

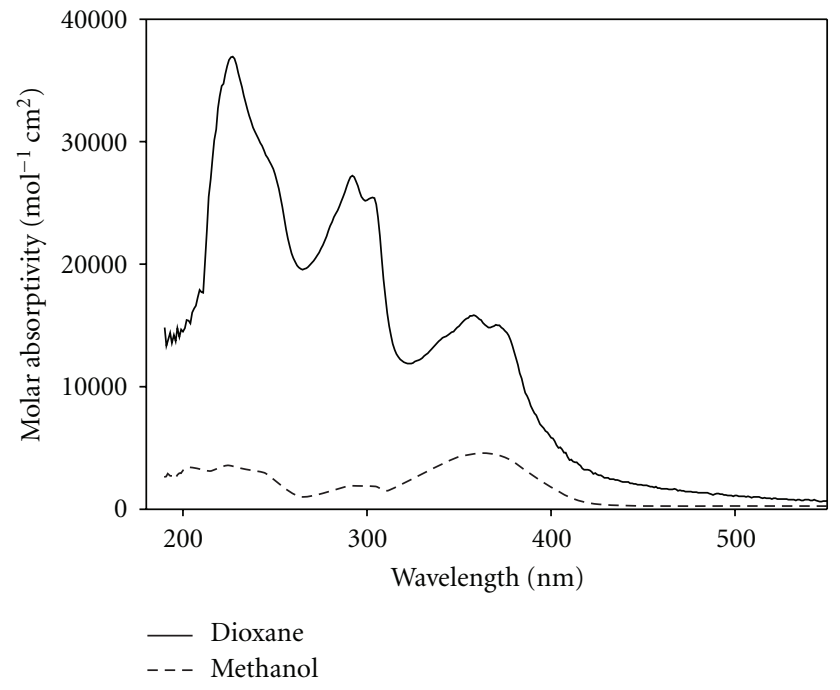

Figure 4: Electronic absorption spectra of (4).
$225 \mathrm{~nm}$. The spectrum, in methanol, is similar to that of dioxane except that the long wavelength band shows a marked red shift of about $13 \mathrm{~nm}$. All observed transitions may be assigned to be $\pi-\pi^{*}$ transition as reflected from their intensities (8000-26000). The effect of $\mathrm{OCH}_{3}$ substitution on the observed spectrum is intensification and slight perturbation of band position, a typical behavior due to its inductive effect.

The long wavelength band computed theoretically centered at $361 \mathrm{~nm}$ is composed of one configuration, $\psi_{62}^{-1} \psi_{63}$, and is assigned as a delocalized band. All the observed transitions are predicted theoretically. The assignments of all transition are listed in Tables 5 and 6.
4.4. The Electronic Absorption Spectra of (4). The methyl and methoxy groups are electron donor groups, whereas $\mathrm{Cl}$-atom is an electron donor by its mesomeric effect and is an electron acceptor by its inductive effect. The electronic spectra of (4) in polar and nonpolar solvents are presented in Figures 4(a) and 4(b). The state energies, assignment, type of electronic transition, theoretically computed and experimentally observed band energies are given in Tables 7 and 8 .

The spectrum in dioxane show six bands centered at $374 \mathrm{~nm}, 355 \mathrm{~nm}, 305 \mathrm{~nm}, 290 \mathrm{~nm}, 250 \mathrm{~nm}$, and $228 \mathrm{~nm}$, whereas the spectrum in methanol shows bands centered at $358 \mathrm{~nm}, 304 \mathrm{~nm}, 298 \mathrm{~nm}, 250 \mathrm{~nm}$, and $227 \mathrm{~nm}$. The band at $374 \mathrm{~nm}$ in dioxane does not appear in methanol due 

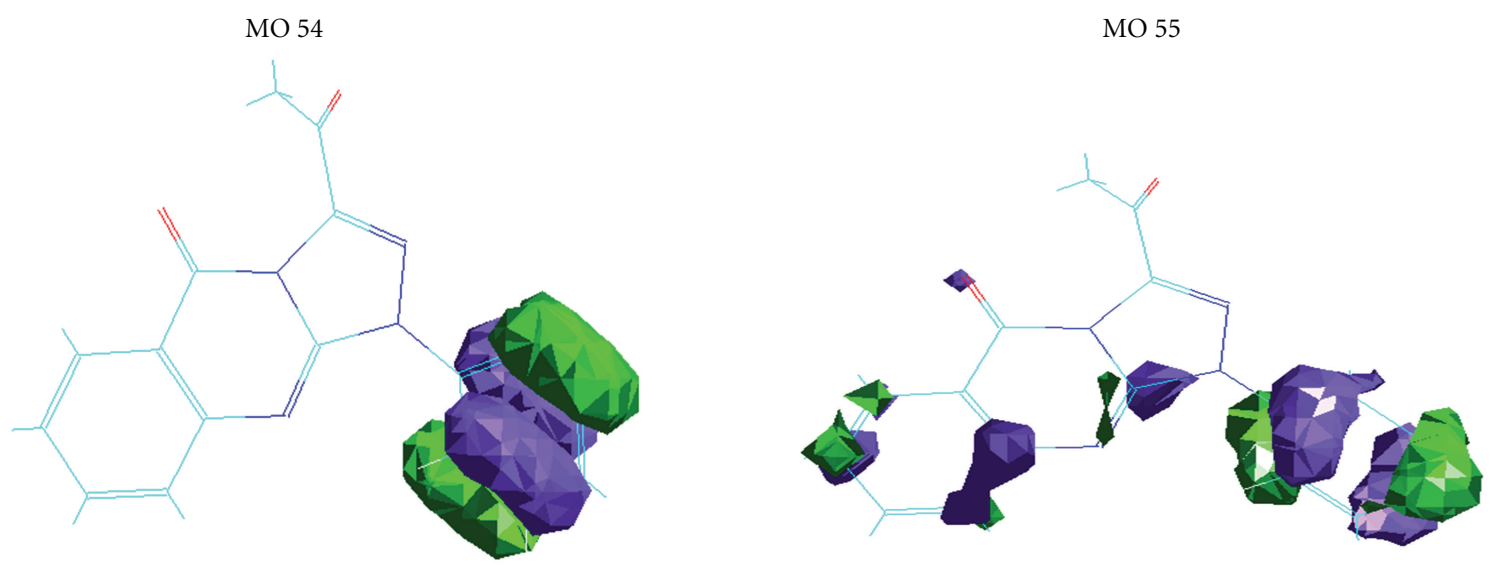

MO 56

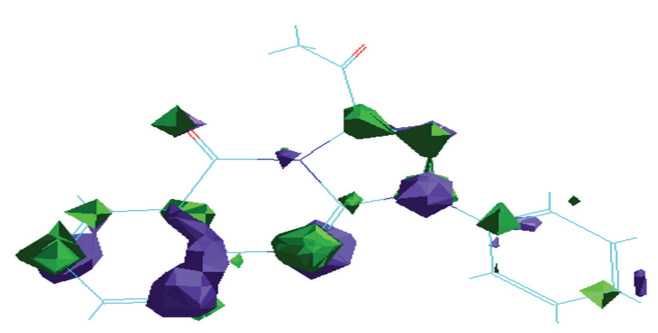

MO 57

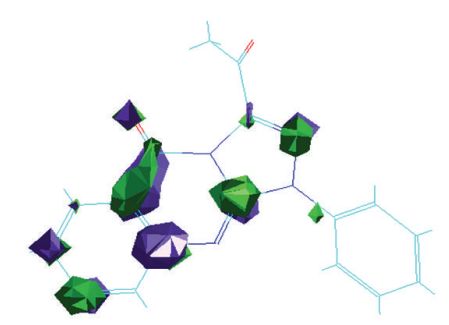

MO 58

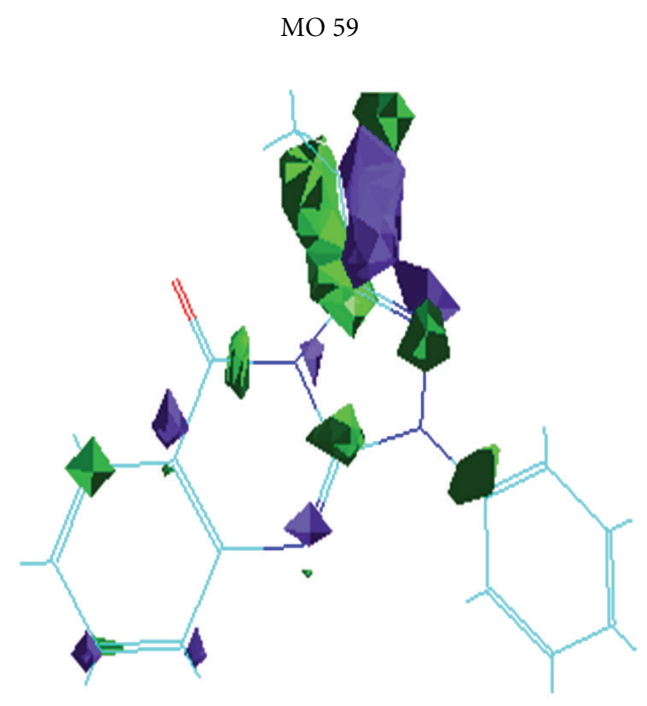

FIgURE 5: The charge density maps of the occupied and vacant MOs for $\mathbf{1}$.

to the low solubility of the compound. Increasing solvent polarity, on going from dioxane to methanol, decreases the intensities of all bands with a red shift of the band at 290 in dioxane to $298 \mathrm{~nm}$ in methanol $(\approx 9 \mathrm{~nm})$. The assignment and coefficient of each configuration in every state are given in Tables 7 and 8.

The long wavelength bands, corresponding to states I, II, and III, are localized and delocalized bands, whereas states 
TABLE 7: Calculated and observed band maxima, intensities, and dipole transition of 3-Acetyl-1-(4-chloro-phenyl)-1H-[1, 2, 4] triazolo [3, 4-b] quinazolin-5-one.

\begin{tabular}{|c|c|c|c|c|c|c|c|}
\hline \multirow{3}{*}{ State } & \multicolumn{5}{|c|}{ Theoretical } & \multicolumn{2}{|c|}{ Experimental } \\
\hline & Configuration & Coefficient & $\lambda, \mathrm{nm}$ & $f$ & $\mu$ & Polar & Nonpolar \\
\hline & & & & & & $\lambda, \mathrm{nm}$ & $\lambda, \mathrm{nm}$ \\
\hline \multirow{2}{*}{ I } & $59-60$ & 0.447 & 377 & 0.001 & 7.3992 & - & 374 \\
\hline & $59-61$ & 0.824 & & & & & \\
\hline II & $59-60$ & 0.894 & 355 & 0.6553 & 3.0741 & 358 & 355 \\
\hline III & $59-61$ & 0.830 & 310 & 0.2924 & 7.0622 & 304 & 305 \\
\hline IV & $59-62$ & 0.919 & 280 & 0.0792 & 4.2378 & 298 & 290 \\
\hline V & $56-60$ & 0.767 & 243 & 0.3583 & 6.4620 & 250 & 250 \\
\hline \multirow{2}{*}{ VI } & $57-60$ & 0.688 & 224 & 0.3585 & 12.0667 & 227 & 228 \\
\hline & $57-62$ & 0.697 & & & & & \\
\hline
\end{tabular}

TABLE 8: State energies, configurations, type of transition, and assignment of all transitions of 3-Acetyl-1-(4-chloro-phenyl)-1H-[1, 2, 4] triazolo [3, 4-b] quinazolin-5-one.

\begin{tabular}{|c|c|c|c|c|c|}
\hline \multirow{2}{*}{ State } & \multirow{2}{*}{ Configuration } & \multirow{2}{*}{ Type } & \multirow{2}{*}{ Assignment } & \multicolumn{2}{|c|}{$\triangle E, \mathrm{eV}$} \\
\hline & & & & Obs. & Calc. \\
\hline \multirow{2}{*}{ I } & $59-60$ & $\pi-\pi^{*}$ & Delocalized band & 3.32 & 3.29 \\
\hline & $59-61$ & $\pi-\pi^{*}$ & Localized band & & \\
\hline II & $59-60$ & $\pi-\pi^{*}$ & Delocalized band & 3.49 & 3.49 \\
\hline III & $59-61$ & $\pi-\pi^{*}$ & Localized band & 4.07 & 4.00 \\
\hline IV & $59-62$ & $\pi-\pi^{*}$ & CT: N- aryl $\rightarrow$ Triazolo-pyrimidine & 4.28 & 4.43 \\
\hline $\mathrm{V}$ & $56-60$ & $\pi-\pi^{*}$ & CT: N-aryl $\rightarrow$ Triazolo-pyrimidine & 4.96 & 5.10 \\
\hline \multirow{2}{*}{ VI } & $57-60$ & $\pi-\pi^{*}$ & CT: N-aryl $\rightarrow$ Triazolo-pyrimidine & 5.44 & 5.54 \\
\hline & $57-62$ & $\pi-\pi^{*}$ & CT: N-aryl $\rightarrow$ Triazolo-pyrimidine & & \\
\hline
\end{tabular}

IV, V, and VI show considerable charge transfer character from $\mathrm{N}$-aryl to triazolopyrimidine.

\section{Summary and Conclusion}

The present analysis of the electronic absorption spectra of the studied Triazolopyrimidines indicates clearly the following.

(1) The spectra are diffuse and reflect the complexity of the molecular forces in this series of molecules. Conjugation extents all over the entire molecular frame work.

(2) The spectra are both solvent and substituent sensitive. Spectra in methanol are, in general, diffuse and less resolved. In case of the parent compound 1, the two long wavelength envelopes do not show significant solvent dependence. On the other hand, the short wavelength band systems show marked red shift by increasing solvent polarity. The origin of this shift can be understood by inspecting the charge density maps for the MOs 56 and 59. The occupied MO 56 is localized to a great extent on the phenyl moiety whereas 59 is localized on the quinazolin subsystem. This charge transfer interaction stabilizes the excited state and thus causes the red shift.
(3) The methyl derivative 2 shows the same behavior as the parent compound $\mathbf{1}$. On the other hand, the methoxy derivative 3 shows the opposite trend. Thus, the two band systems are solvent dependent where a marked blue shift is observed. Furthermore, an increase of band intensity is also marked for the short wave length band system. This behavior can be attributed to $\mathrm{H}$-bonding. This blue shift may indicate a specific solute-solvent interaction due to a hydrogen bond between the oxygen atom of the methoxy group and the alcohol $\mathrm{OH}$ [26]. Jankulovska et al. [27] in their recent analysis of solvatochromic effects pinpoint the need for a complete model and detailed analysis of the effect of protic solvents. The data at hand is not sufficient for such analysis. Theoretical modelling of solvent-solute interaction seems also necessary [28]. Such analysis will be the subject of future study.

(4) Increasing solvent polarity has thus the general effect of diffusing the observed band envelopes and reducing their intensity. This may be attributed to marked solvent-solute interaction rather than just dipolar effect.

(5) Electron-donating substituents, $-\mathrm{CH}_{3}$ and $-\mathrm{OCH}_{3}$, show the one and same trend. The long wavelength 
envelope is diffuse and can only be resolved into one peak. It is interesting to note that the $-\mathrm{CH}_{3}$ derivative shows its long wavelength peak at $376 \mathrm{~nm}$ whereas that for the $-\mathrm{OCH}_{3}$ derivative is at $355 \mathrm{~nm}$. The second band system shows almost the same trend. Only one peak is observed whose position is blue shifted by $12 \mathrm{~nm}$ in case of the $-\mathrm{OCH}_{3}$ as compared to the $-\mathrm{CH}_{3}$ derivative.

\section{References}

[1] A. E. Rashad, M. S. Mohamed, M. E. A. Zaki, and S. S. Fatahala, "Synthesis and biological evaluation of some pyrrolo[2,3-d]pyrimidines," Archiv der Pharmazie, vol. 339, no. 12, pp. 664-669, 2006.

[2] J.-H. Chern, K.-S. Shia, T.-A. Hsu et al., "Design, synthesis, and structure-activity relationships of pyrazolo[3,4d]pyrimidines: a novel class of potent enterovirus inhibitors," Bioorganic and Medicinal Chemistry Letters, vol. 14, no. 10, pp. 2519-2525, 2004.

[3] P. Dolakova, M. Dracinsky, M. Masojidkova, V. Solinova, V. Kasicka, and A. Holy, "Acyclic nucleoside bisphosphonates: synthesis and properties of chiral 2-amino4,6-bis[(phosphonomethoxy)alkoxy]pyrimidines," European Journal of Medicinal Chemistry, vol. 44, no. 6, pp. 2408-2424, 2009.

[4] P. G. Baraldi, M. G. Pavani, M. D. C. Nuez et al., "Antimicrobial and antitumor activity of $\mathrm{n}$-heteroimmine-1,2,3dithiazoles and their transformation in triazolo-, imidazo-, and pyrazolopirimidines," Bioorganic and Medicinal Chemistry, vol. 10, no. 2, pp. 449-456, 2002.

[5] M. N. Nasr and M. M. Gineinah, "Pyrido[2,3-d]pyrimidines and pyrimido $\left[5^{\prime}, 4^{\prime}: 5,6\right]$ pyrido[2,3-d] pyrimidines as new antiviral agents: synthesis and biological activity," Archiv der Pharmazie, vol. 335, no. 6, pp. 289-295, 2002.

[6] Y. M. Loksha, E. B. Pedersen, P. La Colla, and R. Loddo, "Synthesis and Anti-HIV-1 Activity of 1-Substiuted 6-(3Cyanobenzoyl) and [(3-Cyanophenyl)fluoromethyl]-5-ethyluracils," Archiv der Pharmazie, vol. 342, no. 9, pp. 501-506, 2009.

[7] D. Raffa, B. Maggio, F. Plescia et al., "Pyrazolo[3,4d]pyrimidine derivatives as COX-2 selective inhibitors: synthesis and molecular modelling studies," Archiv der Pharmazie, vol. 342, no. 6, pp. 321-326, 2009.

[8] A. A. Aly, "Synthesis and pharmacological activity of annelated pyrimidine derivatives," Phosphorus, Sulfur and Silicon and the Related Elements, vol. 181, no. 6, pp. 1285-1298, 2006.

[9] A. E.-S. Rashad, A. H. Shamroukh, M. I. Hegab, and H. M. Awad, "Synthesis of some biologically active pyrazoles and Cnucleosides," Acta Chimica Slovenica, vol. 52, no. 4, pp. 429434, 2005.

[10] A. R. Katritzky and C. W. Rees, Comprehensive Heterocyclic Chemistry: Pyrimidines, vol. 13, pp. 275-276, 2004.

[11] D. Loakcs, D. M. Brown, and S. A. Salisbury, "A dimroth rearrangement of pyrimidine nucleosides," Journal of the Chemical Society-Perkin Transactions 1, no. 10, pp. 13331337, 1999.

[12] D. Loakes, D. M. Brown, and S. A. Salisbury, "Cyclisation and rearrangement of N-acylaminodeoxycytidines," Tetrahedron Letters, vol. 39, no. 22, pp. 3865-3868, 1998.
[13] T. Nagamatsu and R. Islam, "Regioselective $\mathrm{N}$ - and O-Alkylation of $3 \mathrm{H}-[1,2,3]$ triazolo-[4,5-d]pyrimidine$5,7(4 \mathrm{H}, 6 \mathrm{H})$-diones (8-azaxanthines) and transformation of 3-alkyl derivatives into 1-alkyl isomers," Heterocycles, vol. 68, no. 9, pp. 1811-1820, 2006.

[14] B. M. Lynch, A. J. Robertson, and J. G. K. Webb, "Purine analogues. I. The status of Hückel molecular orbital calculations as predictors of proton shifts, basic strengths, and reactivity," Canadian Journal of Chemistry, vol. 47, no. 7, pp. 1129-1138, 1969.

[15] P. Beltrame, P. L. Beltrame, and M. Simonetta, "Molecular orbital calculations for nucleophilic aromatic substitution reactions in AZA-compounds," Tetrahedron, vol. 24, no. 7, pp. 3043-3051, 1968.

[16] T. Nakajma and A. Pullman, Journal de Chimie Physique, vol. 55, p. 793, 1958.

[17] A. R. Jorge, M. A. Remero, J. M. Salas, M. El-Bahraoui, and J. Molina, Journal of Inorganic Chemistry, vol. 35, pp. 7829-7835, 1966.

[18] A. R. Matecki, Transition Metal Chemistry, vol. 35, p. 801, 2010.

[19] R. Hilal, M. F. Shibl, L. I. Ali, and S. Abdel Halim, submittedto International Journal of Quantum Chemistry.

[20] N. M. Rateb and A. O. Abdelhamid, "Synthesis of 2,3Dihydro-1,3,4-thiadiazole, thiazole, and triazolo[4,3a]pyrimidine derivatives from ethyl benzoylacetate," Heteroatom Chemistry, vol. 15, no. 2, pp. 107-113, 2004.

[21] C. Lee, W. Yang, and R. G. Parr, "Development of the ColleSalvetti correlation-energy formula into a functional of the electron density," Physical Review B, vol. 37, no. 2, pp. 785789, 1988.

[22] A. D. Becke, "Density-functional thermochemistry. III.The role of exact exchange," The Journal of Chemical Physics, vol. 98, no. 7, pp. 5648-5652, 1993.

[23] J. Hasegawa, M. Ishida, T. Nakajima et al., "Gaussian 03, Revision B.01,” Gaussian, Inc., Pittsburgh, Pa, USA, 2003.

[24] L. K. Hanson, J. Fajer, M. A. Thompson, and M. C. Zerner, "Electrochromic effects of charge separation in bacterial photosynthesis: theoretical models," Journal of the American Chemical Society, vol. 109, no. 15, pp. 4728-4730, 1987.

[25] R. Hilal, A. A. Abdel Khalek, and S. A. K. Elroby, "Theoretical and experimental investigation of the electronic spectra of antitumor compounds," Spectroscopy, vol. 20, no. 5, pp. 4253, 2005.

[26] F. Lahmani, E. Bréhéret, and J. Sepio, "Solvation effects of protic solvents on 9-methoxyanthracene in a supersonic free jet," Journal of Photochemistry and Photobiology, A: Chemistry, vol. 62, no. 1, pp. 33-44, 1991.

[27] M. Jankulovska, L. Šoptrajanova, I. Spirevska, K. Čolančevska Ragenovi, and S. Ristovski, "Investigation of solvent effects on electronic absorption spectra of some substituted 1,2,4triazoline-3-thiones," Macedonian Journal of Chemistry and Chemical Engineering, vol. 29, no. 1, pp. 43-50, 2010.

[28] T. Maeyama, K. Yoshida, I. Yagi, A. Fujii, and N. Mikami, "Interpreting the physical background of empirical solvent polarity via photodetachment spectroscopy of microsolvated aromatic ketyl anions," Journal of Physical Chemistry A, vol. 113, no. 40, pp. 10593-10602, 2009. 


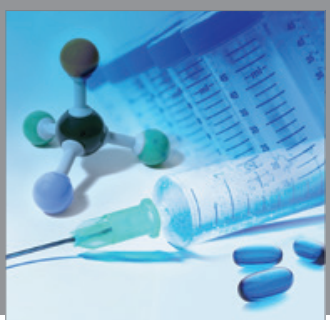

International Journal of

Medicinal Chemistry

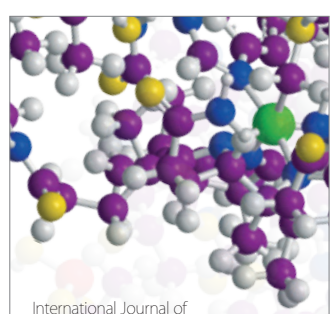

Carbohydrate Chemistry

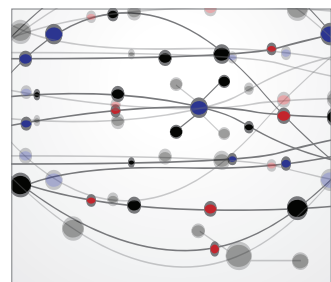

The Scientific World Journal
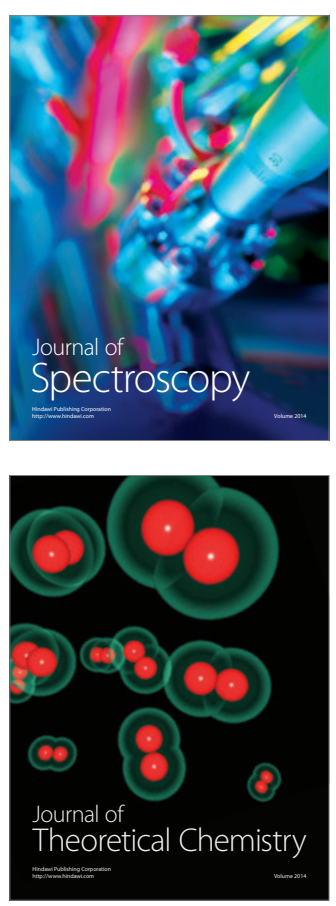
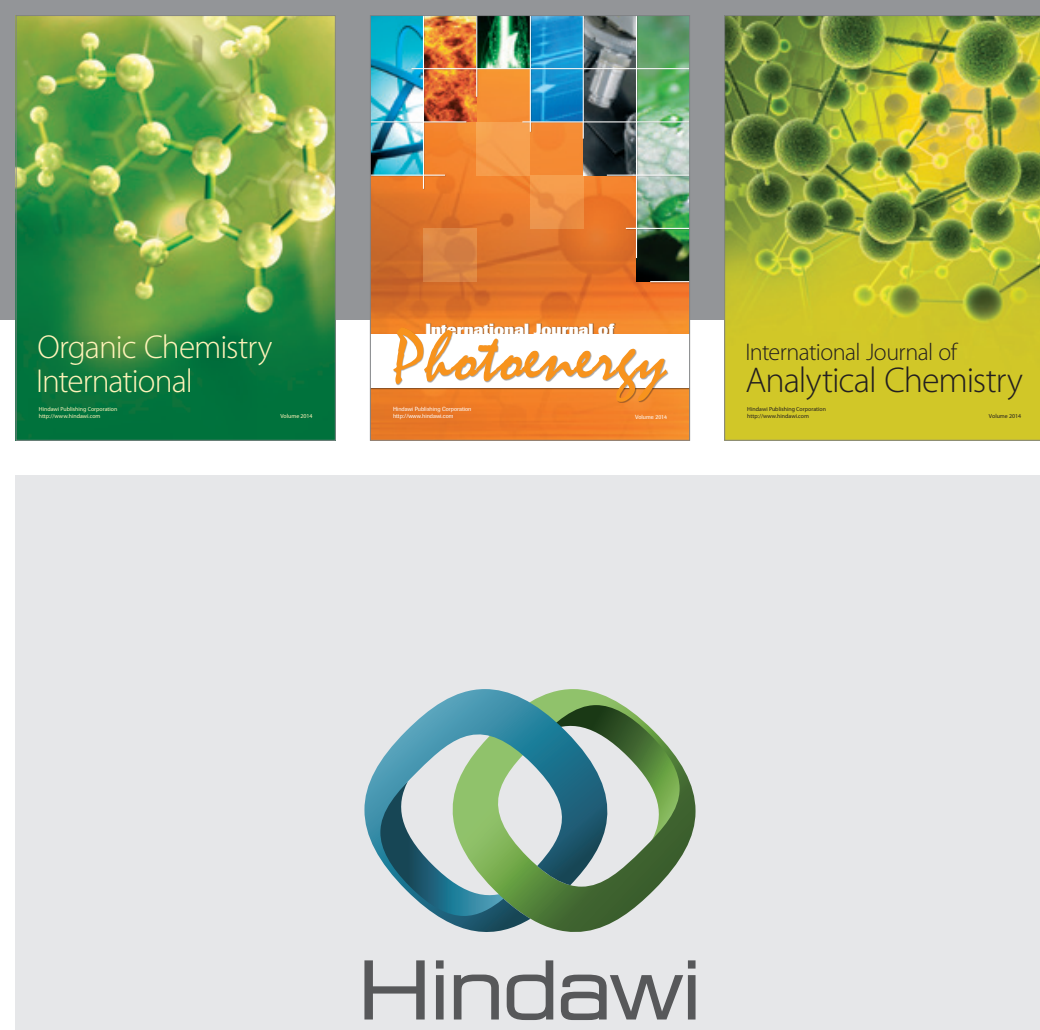

Submit your manuscripts at

http://www.hindawi.com
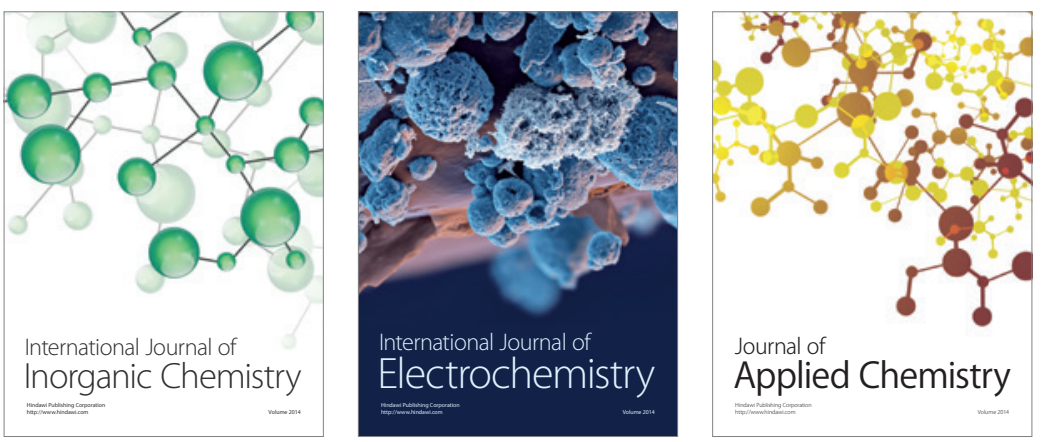

Journal of

Applied Chemistry
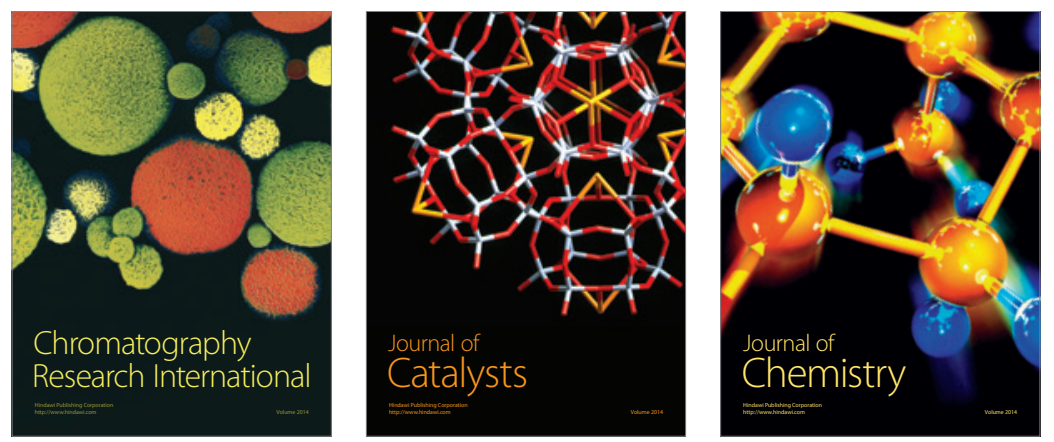
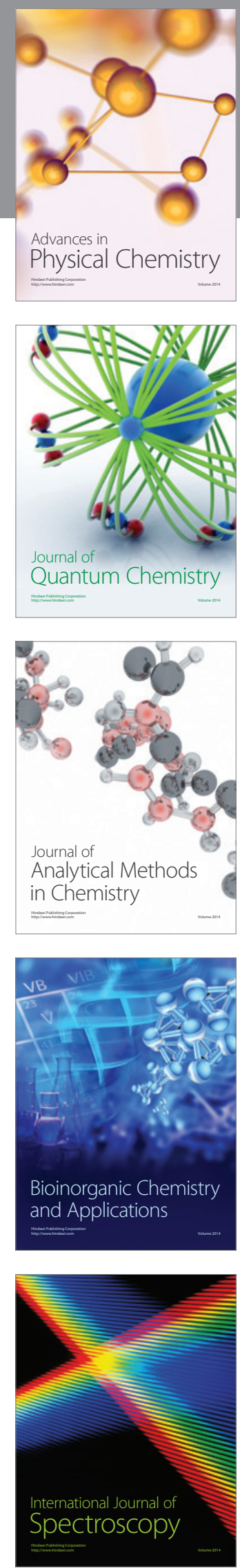